\title{
PREOPERATIVE EVALUATION OF OVARIAN TUMORS IN THE RURAL POPULATION- CLINICAL FEATURES, GREY SCALE ULTRASOUND, COLOUR DOPPLER, SERUM CA-125 AND ALCAZAR SCORE IN PREDICTING MALIGNANCY
}

Ananya Sinha, Chella Hariharan, Rahul K Chaudhary
1. Junior Resident. Department of Obstetrics \& Gynaecology, Jawaharlal Nehru Medical College, Acharya Vinoba Bhabhe Rural Hospital, Sawangi (Meghe), Wardha.
2. Professor \& Head. Department of Obstetrics \& Gynaecology, Jawaharlal Nehru Medical College, Acharya Vinoba Bhabhe Rural Hospital, Sawangi (Meghe), Wardha.
3. Assistant Professor. Department of General Surgery, Jawaharlal Nehru Medical College, Acharya Vinoba Bhabhe Rural Hospital, Sawangi (Meghe), Wardha.

\section{CORRESPONDING AUTHOR:}

Dr Rahul K Chaudhary,

Flat no 7, Shajida building (near SBI),

Near AVB Rural Hospital, Paloti Road,

Sawangi (Meghe), Wardha- 442001, Maharashtra.

E-mail: rahulkchaudhary@rediffmail.com

\begin{abstract}
PURPOSE: Preoperative diagnosis of early ovarian malignancy is challenging. We evaluated clinical features and the performance of serum CA 125, ultrasonography, colour Doppler, and Alcazar score in diagnosing ovarian cancer. METHODS: This prospective study included 67 patients. Sono-morphological parameters studied were tumor size, bilaterality, wall irregularity, presence of solid component, septae, septae $>3 \mathrm{~mm}$ and ascites. Doppler parameters studied were blood flow, central vascularity, resistance index (RI) $<0.4$, peak systolic velocity (PSV) $>15 \mathrm{~cm} / \mathrm{sec}$ and velocimetry. Alcazar score was calculated. Findings on imaging were correlated with histopathology using univariate analysis. Receiver operator characteristic (ROC) curve of Alcazar score was plotted. RESULTS: Of 67 patients, 14 had malignant disease. Clinical features were not reliable in predicting malignancy. Sonomorphological features- irregular wall, septae $>3 \mathrm{~mm}$, solid areas, mixed/heterogenous echotexture, bilaterality and ascites significantly correlated with malignancy while tumor size did not. On Doppler; increased blood flow, central vascularity and PSV $>15 \mathrm{~cm} / \mathrm{sec}$ significantly correlated with malignancy while RI $<0.4$ did not. The ROC for Alcazar score revealed an AUC of 0.95 and that of CA125 of 0.90 . CONCLUSION : Ultrasound with Doppler as a part of a scoring system (Alcazar score) to diagnose ovarian malignancy is easy to apply and is fairly accurate. CA 125 has low specificity.
\end{abstract}

KEYWORDS: ovarian tumor, ultrasound, Doppler, Alcazar score, CA 125

\author{
ABBREVIATIONS USED \\ AUC: Area Under Curve \\ CA125: Carcinoma Antigen 125 \\ NPV: Negative Predictive Value \\ PI: Pulsatility Index \\ PPV: Positive Predictive Value \\ PSV: Peak Systolic Velocity \\ RI: Resistance Index
}


INTRODUCTION: An adnexal mass is a common occurrence in clinical practice. The differential diagnosis varies from functional cyst to benign and malignant tumors of the ovary. ${ }^{1}$

Epithelial ovarian cancer is the most common malignancy of ovary and greater than $70 \%$ patients present during the third or fourth stage. This is because most of these patients do not experience any signs or symptoms until the disease spreads to the upper abdomen. ${ }^{2}$

Early identification of a lurking malignancy is of paramount importance as early stage ovarian malignancy carries a more favourable prognosis. ${ }^{2}$ This warrants a high index of suspicion. A percutaneous biopsy is not recommended during the initial evaluation as this can result in cyst rupture and spillage of malignant cells into the peritoneal cavity. ${ }^{3}$ This emphasizes the fact that the clinician has to arrive at reasonably accurate diagnosis using non-invasive techniques to avoid an unnecessary laparotomy. Till date there is no universally accepted test or criteria to exclude cancer with certainty. ${ }^{4}$ And often, surgery is required to solely exclude the possibility of a malignancy. About one-third of tumors operated upon for suspected ovarian cancer turn out to benign. ${ }^{5}$ Age, menopausal status, family history of cancer may increase our suspicion. Clinical features do not help much with early lesions.

Serum levels of CA 125 are elevated ( $>35 \mathrm{U} / \mathrm{mL}$ ) in $82 \%$ of patients with epithelial ovarian tumors. ${ }^{2}$ However, it is not a reliable diagnostic test as it is also elevated in a variety of benign gynaecological conditions like endometriosis, pelvic inflammatory disease or pregnancy and non gynaecological malignancies (such as breast, lung and gastrointestinal cancers). ${ }^{3}$ Furthermore, it is elevated only in approximately $50 \%$ of patients with early ovarian cancer. Its uses are mainly restricted to assess response to therapy and detection of relapse.

Ultrasonography is a readily available, non invasive modality for evaluating ovarian tumors and has a high negative predictive value. ${ }^{6}$ The sensitivity and specificity of ultrasonography in differentiating a benign from a malignant ovarian tumor is $82 \%$ and $92 \%$ respectively. ${ }^{7}$ However, it is highly operator dependant and the extremely variable morphological characteristics of an ovarian neoplasm makes a precise diagnosis difficult when this modality is used alone.

The classic sonographic finding of malignancy is a "complex" cyst, defined as containing both solid and cystic components, sometimes with septations and internal echogenecity. ${ }^{2}$ Presence of papillary structures, irregular inner walls and bilateral masses are again more common with malignancy. In contrast, a "simple cyst" is defined as being thin walled, being fluid filled, without a mass component, septations or internal echogenicity. These tumors are usually benign. Nevertheless, treatment has to be individualized. For example, a postmenopausal lady with a simple cyst that exceeds $10 \mathrm{~cm}$ in diameter and with a raised serum CA 125 would be referred for surgery. ${ }^{2}$

Colour and pulsed Doppler sonography have been advocated for distinguishing benign from malignant ovarian masses. ${ }^{8}$ It is based on the premise that malignant masses will have higher diastolic flow due to neovascularization that can be detected on spectral waveforms. Malignant tumor growth is dependent on angiogenesis, but these tumor vessels are abnormal as they lack smooth muscle in their walls. This, along with arteriovenous shunting, leads to decreased vascular resistance and thus, higher diastolic flow velocity.

The pulsatility index (PI) and the resistive index (RI) have been mostly used to analyze the Doppler waveform pattern. The PI is the peak systolic velocity minus the end diastolic velocity divided by the mean velocity; the RI is the peak systolic velocity minus the end diastolic velocity divided by peak systolic velocity. Malignant ovarian masses usually have a PI $<1$ or an RI $<0.4$. The lowest PI or RI obtained is considered. Vessel location also helps in Doppler 
assessment with malignant lesions having more central flow and benign lesions more peripheral. ${ }^{8}$

There have been various scoring systems based on morphology on ultrasound imaging designed to determine the risk of an ovarian tumor being malignant. The most common systems published are those given by Granberg et al, Sassone et al, De Priest at, Lerner et al and the "Multicentre" scoring system.9-12 These systems have a high sensitivity and have served to reduce interobserver variability. However, a completely reliable differentiation of benign from malignant masses cannot be obtained from morphological criteria alone.

Similarly, scoring systems have been developed for Doppler sonography - the "vascular" score as described by Caruso et al.13 Studies have shown that Doppler, when added to sonographic morphologic assessment, improves specificity and positive predictive value. Multiparameter scoring systems have been proposed by Brown et al and more recently by Alcazar et al incorporating both morphologic assessment and Doppler features.8,14,15 (table 1)

The number of scoring systems available only serve to emphasize that we are dealing with an imprecise tool. Considering that most patients with epithelial ovarian cancer present in advanced stage $(>70 \%)$, we need to have an accurate diagnostic test. ${ }^{2}$ In this study, we will evaluate patients with ovarian masses with ultrasonography, Doppler and CA 125 preoperatively. We will use a multiparameter (ultrasound + Doppler) score- that proposed by Alcazar et al and assess its ability in predicting underlying malignancy. ${ }^{15,16}$

METHODS: This prospective study included all patients with ovarian tumor who were diagnosed with a pelvic mass that was suspected to be of ovarian origin and were expected to undergo surgery at a reputed tertiary rural hospital between April 2010 and July 2012.

Patients who had already undergone surgery for ovarian tumor or in whom clinical as well as investigational parameters were insufficient were excluded from the study. Patients in whom a histopathological diagnosis was not arrived at were excluded.

A detailed history and complete physical examination of patients with ovarian mass was done. All patients were evaluated with ultrasonography abdomen / transvaginal ultrasound and colour Doppler study (if affording), by Junior Consulting Radiologists at the Department of Radiology at the tertiary center.

The Phillips Envisor ultrasound colour Doppler unit was used to evaluate patients. Trans-abdominal sonography was done and if necessary, transvaginal sonography was done for clearer evaluation.

Ultrasonographic parameters that were studied were tumor volume, inner wall structure, wall thickness, septations, solid area (absence or presence of any solid area $\geq 1 \times 1 \mathrm{~cm}$ in the internal wall surface of the septum) and echogenicity (cystic, anechoic, homogenous content, heterogenous content or purely solid).

Colour Doppler parameters that were studied include blood flow (present or absent), amount of blood flow (scant, moderate, abundant), blood flow location (central or peripheral), RI (resistance index) and PSV (peak systolic velocity)

Patients were then assigned a score as per the scoring system by Alcazar et al. (table 1) Serum CA 125 was determined.(preoperative) All patients who were then subjected to surgery or if a pathological diagnosis was obtained (as in presence of malignant cells in ascitic fluid) were included in the study. The remaining were excluded. 
The age distribution as well as the pathological types of ovarian tumor were studied. Clinical features were studied and their correlation with specific pathological tumor type were studied. Findings on investigation were correlated with histopathological findings.

The various parameters studied in ultrasound and Doppler were analyzed for their ability to accurately predict the diagnosis of a malignancy. Serum CA 125 levels were studied similarly for its correlation with histopathology. The Alcazar score was evaluated for its ability to accurately diagnose malignancy.

Data was tabulated using Microsoft Excel 2007. Statistical analysis of the data was done on SPSS computer statistics program(version 16) and MedCalc. References were tabulated as per the Vancouver system.

RESULTS: This study included 67 patients. Eleven patients were suffering from epithelial ovarian cancer, two from a sex cord stromal tumor and one from germ cell tumor. The remaining patients had a benign pathology.(table 2)

The age of patients ranged from 11 to 73 years. Most patients with a benign tumor belonged to the 31 to $40 \mathrm{yr}$ age group. Patients with a malignant lesion were mostly greater than 40 years of age. $(p<0.05)$

Among 14 patients with malignant disease, only four had early stage cancer (stage I disease). Two patients had stage II disease, four stage III and four patients stage IV disease.

Non specific symptoms like constipation, weight loss, early satiety/ anorexia were more common in patients with malignancy. Abdominal distention and pleural effusion were more commonly seen in patients with a malignancy. Other symptoms like abdominal pain, lump were not helpful in differentiating benign from a malignant mass.

Twenty one patients (31\%) were postmenopausal. Thirty eight percent of lesions in postmenopausal patients were malignant as compared to only $13 \%$ in premenopausal patients. $(p<0.05)$

Univariate analysis of variables assessed by ultrasound of ovarian lesions showed that presence of bilateral disease, irregular walls, thick septae $(>3 \mathrm{~mm})$, presence of solid component and mixed/heterogenous echogenicity were significantly associated with the presence of a malignant lesion. Presence of septate lesion and tumor diameter $>5 \mathrm{~cm}$ or even $>10 \mathrm{~cm}$ were not significantly associated with the presence of malignancy. (table 3)

Tumor diameter $>5 \mathrm{~cm}$ though being a very sensitive parameter (sensitivity $90 \%$ ) had a low specificity (15\%). The specificity was improved to $56 \%$ by increasing the cut off to $>10 \mathrm{~cm}$, but at the cost of decreasing the sensitivity to 67\%. (table 4)

Presence of irregular walls, ascites and mixed or heterogenous echotexture of the mass had high sensitivity and specificity rates in predicting malignancy. Presence of bilateral disease had a high specificity but a low sensitivity in predicting malignancy.(table 4)

Doppler evaluation was done for 22 patients. Univariate analysis of colour Doppler parameters showed that abundant to moderate blood flow, central vascularity and peak systolic velocity $>15 \mathrm{~cm} / \mathrm{s}$ were highly predictive of a malignant lesion. $(p<0.0001)$. Resistance index (RI) $<0.4$ did not significantly correlate with the presence of a malignant lesion and had a low specificity (table 3,4; figure 1)

The Alcazar score was evaluated for a total of 19 patients. A score $>=6$ was considered as the cut off for malignancy. The score had a sensitivity of $100 \%$ and a specificity of $91 \%$. The Receiver Operator Characteristic (ROC) for the Alcazar score shows an area under curve of 0.95.(figure 2) 
Serum CA 125 was available for 30 patients. A value of $>35 \mathrm{U} / \mathrm{ml}$ was used as a cut-off criterion of malignancy. It had a high sensitivity of $92.5 \%$, but a specificity of only $83 \%$. Three patients out of 14 patients in whom CA 125 was elevated had benign lesion. The Receiver Operator Characteristic (ROC) for CA 125 in our study shows an AUC of 0.90.(figure 3)

DISCUSSION: Early diagnosis of ovarian cancer has challenged the physician since decades. The absence of a suitable screening test is also a matter of concern as when symptoms do occur, the disease is usually advanced. In our study, too, we had a higher incidence of stage III and stage IV cancers. Only two out of 11 epithelial malignancies were stage 1 . Germ cell tumors and sex cord stromal tumors presented in earlier stages.

Ovarian cancer is usually a disease of older women and in this study too, we found that all epithelial ovarian cancers were confined to women of age $\geq 40$ years of age $(p<0.05)$. This correlated well with the findings of the study by Singh et al and VanGorp et al.16,17 Germ cell and stromal tumors occurred in the younger age group. Maheshwari et alhave reported the highest incidence of benign ovarian tumors in the 20 to 40 year age group. ${ }^{18}$ Our study had similar findings. Twenty six out of 53 benign patients belonged to this age group.

Menopausal status was significantly associated with malignancy. Singh et al and Jacob et al have reported similar findings. ${ }^{16,19}$ In fact, menopausal index is part of the RMI score in predicting malignancy in a patient with an adnexal mass as devised by Jacob et al. ${ }^{19}$

Patients with early ovarian cancer may be asymptomatic or present with non-specific symptoms. In our study, complaints like early satiety, weight loss, constipation and abdominal distention or bloating were more common in patients with malignancy. Urinary disturbances were also more common in cancer patients. Goff et al have reported similar findings. ${ }^{20}$ Complaints of abdominal pain or lump were not helpful in distinguishing a benign from a malignant presentation. However, pain as an acute symptom has been regarded as a symptom of a benign tumor as in torsion. Patients with vomiting also had a benign histology. Patients with infertility had a benign histology. The patients with post-menopausal bleed, irregular menses were too few to derive any conclusion. One patient with stromal tumor presented with virilisation which was due to testosterone production by the tumor.

Presence of ascites and pleural effusion were pointers of malignancy. However, both are pointers of advanced disease. Presence of abdominal lump, a firm to hard mass palpated per vaginally were inconsistently associated with malignant pathology $(p>0.05)$. Thus, there are no reliable features on clinical examination that can differentiate benign from malignant neoplasms in early ovarian cancer. Castillo et al reported that $88 \%$ of ovarian cysts were missed by clinical examination. ${ }^{21}$ Miller et al have reported physician assessment to have a sensitivity of $75 \%$ and specificity of $79 \%$ in predicting ovarian malignancy. ${ }^{20}$

Conventional ultrasound or grey scale ultrasound performs reasonably well as a first investigation for evaluation of the adnexal mass. In our study, we found presence of irregular walls, thick septae $(>3 \mathrm{~mm})$, presence of solid component or mixed echotexture to be significantly correlated with a malignant adnexal mass on univariate analysis.(table 3) Comparison of our findings with those of Singh et al and Timmerman et al show somewhat greater sensitivity and specificity of ultrasound in our study. ${ }^{16,22}$ (table 4) Perhaps the greater percentage of patients with advanced stage tumors and less number of patients included in our study could explain these findings.

We did not find tumor size to significantly correlate with malignant disease though studies by Singh et al and Timmerman et al have found tumor volume greater than $100 \mathrm{ml}$ and 
$200 \mathrm{ml}$ respectively to correlate significantly with malignancy. ${ }^{16,22} \mathrm{~A}$ higher cut off criteria of malignancy of size greater than $10 \mathrm{~cm}$, raised the specificity to $55 \%$ but at the cost of decreased sensitivity (67\%).

Presence of septae did not correlate significantly with the presence of malignancy. Our findings were in contrary to that of Brown et al in this regard.23 On colour Doppler evaluation, we found increased blood flow (abundant and moderate), central vascularity and peak systolic velocity $>15 \mathrm{~cm} /$ second to be significantly associated with malignancy. Singh et al, Timmerman et al, Carter et al, Madan et al and Hossain et al have reported similar findings. 16,22,24-26

Again, the sensitivity and specificity achieved were higher than that reported in literature. This was due to a higher percentage of patients with stage III and IV disease in our study.

Unlike Singh et al we did not find a resistance index $<0.45$ to significantly predict malignancy. ${ }^{16}$ There is a controversy with regard to the use of impedance values (resistance index and pulsatility index) in literature. Valentin et al reported similar findings and concluded that blood flow velocity and tumor colour was a better predictor of malignancy. ${ }^{27}$ Several authors have concluded that Doppler evaluation should not be used alone in evaluation of adnexal massesshould be used in conjunction with sonography. $16,24,28,29$

In general scores based on morphological and Doppler findings fare better than those based on morphology alone. As not all parameters were available in all patients and Doppler carried out in only 22 patients, this score could be evaluated in only 19 patients. We found an Alcazar score of greater than 6 to be highly sensitive and specific for malignancy with a very high area under curve (AUC $=0.95, p<0.0001$ ). (figure 1 ) Our sensitivity and specificity values are similar to those reported by Alcazar et al $^{15}$ and are much better than reported by studies based on morphology alone. . $^{7-12,25}$

Serum CA 125 values were available for 30 patients. Out of 12 patients with malignancy, 11 had this parameter elevated $(>35 \mathrm{U} / \mathrm{ml})$. However, it had a lower specificity and positive predictive value ( $83 \%$ and $78 \%$ respectively). We compared our findings to those of Dodge et al, Myer et al and Hossain et al and found better sensitivity and specificity rates.4,20,26 As there were fewer patients with early stage epithelial ovarian cancer, this test was more accurate in our study.

Recent literature stress the importance of subjective assessment using ultrasound and Doppler (even gray scale ultrasound) in the diagnosis of adnexal masses.,17 Experienced sonologists consider the demographic and clinical profile of patients while assessing ultrasonographic parameters when they estimate the risk of malignancy in an adnexal mass, and they subconsciously apply their knowledge based on their previous observations. This skill is not easily transferrable to less experienced ultrasound examiners. VanGorp et al have reported an AUC of 0.968 for subjective assessment. ${ }^{17}$

Subjective assessment is influenced by experience, but this should not discourage sonologists in non expert centers in the performance of this test for diagnosis or referral of patients with suspected malignancy. This study was carried out at a rural tertiary set up without requirement of expensive equipment and excessive financial burden on patients.

Combining sono-morphological and Doppler assessment has a high sensitivity and specificity in detection of malignant masses of ovary as demonstrated by using the Alcazar score. With continuing improvement of ultrasound equipment recent literature report more accurate diagnostic performance of this modality. A serum marker with high sensitivity and specificity for diagnosis of early epithelial ovarian cancer is still elusive. 
DISCLOSURE STATEMENT / CONFLICT OF INTEREST: None declared.

\section{REFERENCES:}

1. Davies AP, Jacob I, Woolas R, Fish A, Oram D. The adnexal mass: benign or malignant? Evaluation of a risk of malignancy index. Br J Obstet Gynaecol 1993;100:927-31.

2. Cannistra SA, Gershenson DM, Recht A. Ovarian cancer, fallopian tube carcinoma and peritoneal carcinoma. In: DeVita VT, Lawrence TS, Rosenberg SA, DePinho RA, Weinberg RA. DeVita, Hellman and Rosenberg's Cancer: Principles and Practice of Oncology. Vol 2;8th ed. Philadelphia PA, Lippincott Williams and Wilkins 2008; pp 1568-9.

3. Eagle K, Ledermann JA. Tumor Markers in Ovarian Malignancies. Oncologist 1997;2: 324-9.

4. Dodge JE, Covens AL, Lacchetti C, Elit LM, Le T, M Devries-Aboud M, et al. Management of a suspicious adnexal mass: a clinical practice guideline. Curr Oncol. 2012;19:e244-57.

5. D’Arcy J, Jayaram V, Lynch M, Soutter WP, Cosqrove DO, Harvey CJ, et al. Ovarian Cancer detected non-invasively by contrast-enhanced power Doppler ultrasound. BJOG 2004;111:619-22.

6. Jeong YY, Outwater EK, Kang HK. Imaging evaluation of ovarian masses. Radiographics 2000;20:1445-70.

7. Granberg S, Norstrom A, Wikland M. Tumors in the lower pelvis as imaged by vaginal sonography. Gynaec Oncol 1990.37:224-9.

8. Salem S. Gynecology. In: Rumack CM, Wilson SR, Charboneau JW. Diagnostic Ultrasound. Vol 1.3 ${ }^{\text {rd }}$ edition. Philadelphia PA: Elsevier Mosby 2009.pp 563-6.

9. Sassone AM, Timor-Tritsch IE, Artner A, Westhoff C, Warren WB. Transvaginal sonographic characterization of ovarian disease. Evaluation of new scoring system to predict ovarian malignancy. Obstet Gynecol 1991;78:70-6.

10. DePriest PD, Shenson D, Fried A, Hunter JE, Andrews SJ, Gallion HH, et al. A morphology index based on sonographic findings in ovarian cancer. Gynecol Oncol 1993;51:7-11.

11. Lerner JP, Timor-Tritsch IE, Federman A, Abramovich G. Transvaginal ultrasonographic characterization of ovarian masses with an improved, weighted scoring system. Am J Obstet Gynecol 1994;170:81-5.

12. Ferrazzi E, Zanetta G, Dordoni D, Berlanda N, Mezzopane R, Lissoni AA. Transvaginal ultrasonographic characterization of ovarian masses: comparison of five scoring systems in a multicentre study. Ultrasound Obstet Gynecol 1997;10:192-7.

13. Caruso A, Caforio L, Testa AC, Caiampelli M, Panici PB, Mancuso S. Transvaginal color Doppler sonography in the presurgical characterization of adnexal masses. Gynecol Oncol 1996;63:184-91.

14. Buy JN, Ghossain MA, Hugol D, Hassen K, Sciot C, Truc JB, et al. Characterization of adnexal masses: combination of color Doppler and conventional sonography compared with spectral Doppler analysis alone and conventional sonography alone. AJR Am J Roentgenol 1996; 166:385-93.

15. Alcazar JL, Merce LT, Laparte C, Jurado M, Lopez-Garcia C. A new scoring system to differentiate benign from malignant adnexal masses. Am J Obstet Gynecol 2003;188:685-92.

16. Singh U, Neera K, Ekta N. Evaluation of new scoring system to differentiate between benign and malignant adnexal mass. Obstet Gynecol India 2006;56:162-5. 
17. Van Gorp T, Veldman J, Van Calster B, Cadron I, Leunen K, Amant F, et al. Subjective assessment by ultrasound is superior to the risk of malignancy index (RMI) or the risk of ovarian malignancy algorithm (ROMA) in discriminating benign from malignant adnexal masses. Eur J Cancer 2012;48:1649-56.

18. Maheshwari V, Tyagi SV, Saxena K, Tyagi N, Sharma R, Aziz M, et al. Surface epithelial tumors of ovary. Indian J Pathol Microbiol 1994;37:75-85.

19. Jacobs I, Oram D, Fairbanks J, Turner J, Frost C, Grudzinskas JG. A risk of malignancy index incorporating CA 125, ultrasound and menopausal status for the accurate preoperative diagnosis of ovarian cancer. Br J Obstet Gynaecol. 1990;97:922-9.

20. Miller RW, Ueland FR. Risk of Malignancy in Sonographically Confirmed Ovarian Tumors. Clin Obstet Gynecol 2012;55:52-64.

21. Castillo G, Alcazar JL, Jurado M. Natural history of sonographically detected simple unilocular adnexal cysts in asymptomatic postmenopausal women. Gynecol Oncol 2004; 92:965-9.

22. Timmerman D, Testa AC, Bourne T, Ameye L, Jurkovic D, Van Holsbeke C, et al. Simple ultrasoundbased rules for the diagnosis of ovarian cancer. Ultrasound Obstet Gynecol 2008;31:681-90.

23. Brown DL, Doubilet PM, Miller FH, Frates MC, Laing FC, DiSalvo DN, et al. Benign and malignant ovarian masses: Selection of the most discriminating gray-scale and Doppler sonographic features. Radiology 1998;208:103-10.

24. Carter J, Saltzman A, Hartenbach E, Fowler J, Carson L, Twiggs LB. Flow characteristics in benign and malignant gynaecologic tumors using transvaginal colour flow Doppler. Obstet Gynecol 1994;83:125-30.

25. Madan R, Narula MK, Chitra R, Bajaj P. Sonomorphological and Color Doppler flow imaging evaluation of adnexal masses. Indian J Radiol Imaging 2004;14:365-72.

26. Hossain F, Karim MN, Rahman SM, Khan N, Siddiqui M, Hussain R. Preoperative detection of ovarian cancer by color Doppler ultrasonography and CA 125. Bangladesh Med Res Counc Bull. $2010 ; 36: 68-73$.

27. Karlsen MA, Sandhu N, Hogdall C, Christensen IJ, Nedergaard L, Lundvall L, et al. Evaluation of HE4, CA125, risk of ovarian malignancy algorithm (ROMA) and risk of malignancy index (RMI) as diagnostic tools of epithelial ovarian cancer in patients with a pelvic mass. Gynecol Oncol 2012;127:379-83.

28. Schelling M, Braun M, Kuhn W, Bogner G, Gruber R, Gnirs J, et al. Combined transvaginal B-mode and color Doppler sonography for differential diagnosis of ovarian tumors: results of a multivariate logistic regression analysis. Gynecol Oncol 2000;77:78-86.

29. Guerriero S, Ajossa S, Risalvato A, Lai MP, Mais V, Angiolucci M, et al. Diagnosis of adnexal malignancies by using colour Doppler energy imaging as a secondary test in persistent masses. Ultrasound Obstet Gynecol 1998;11:277-82. 
TABLE 1: The Alcazar Score 15

\begin{tabular}{|c|c|c|c|c|}
\hline score & $\begin{array}{c}\text { Thick papillary } \\
\text { projection }\end{array}$ & $\begin{array}{c}\text { Solid areas or purely sold } \\
\text { echogenicity }\end{array}$ & $\begin{array}{c}\text { Blood flow } \\
\text { location }\end{array}$ & Velocimetry \\
\hline 0 & absent & absent & $\begin{array}{c}\text { Absent or } \\
\text { peripheral }\end{array}$ & Other \\
\hline 2 & Present & - & - & $\begin{array}{c}\text { High velocity/ low } \\
\text { resistance }\end{array}$ \\
\hline 4 & - & present & central & - \\
\hline
\end{tabular}

TABLE 2: Histology of tumors included in the study

\begin{tabular}{|c|c|}
\hline pathology & No of patients \\
\hline Epithelial malignancy & $11)$ \\
\hline Sex cord stromal tumor & $\succ 14$ \\
\hline Germ cell tumor & $1 J$ \\
\hline Simple serous cyst & 10 \\
\hline Serous cystadenoma & 9 \\
\hline Mucinous cystadenoma & 4 \\
\hline Endometrioma & 4 \\
\hline Dermoid cyst & 4 \\
\hline cystadenofibroma & 1 \\
\hline Benign (unspecified) & 2 \\
\hline Hemorrhagic ovarian cyst & 16 \\
\hline Simple mucinous cyst & 1 \\
\hline Tuberculosis & 1 \\
\hline
\end{tabular}


TABLE 3: Variables used for sono-morphological and Colour Doppler assessment of ovarian masses- their presence in patients with benign and malignant lesions and univariate analysis

\begin{tabular}{|c|c|c|c|}
\hline Ultrasonic Parameters & $\begin{array}{l}\text { No of patients with } \\
\text { Malignant tumor }\end{array}$ & $\begin{array}{l}\text { No of patients with } \\
\text { Benign tumor }\end{array}$ & $p$-value \\
\hline Tumor Diameter $>5 \mathrm{~cm},{ }^{*} \mathrm{n}=57$ & $11(91.6 \%) \dagger$ & $38(84.4 \%)^{\ddagger}$ & 0.50 \\
\hline Tumour diameter $>10 \mathrm{~cm}, \mathrm{n}=57$ & $8(66.7 \%)$ & $20(44.4 \%)$ & 0.06 \\
\hline Irregular wall, $\mathrm{n}=38$ & $6(100 \%)$ & $1(3.1 \%)$ & $<0.0001$ \\
\hline Bilateral Disease, $\mathrm{n}=54$ & $6(54.5 \%)$ & $4(9.3 \%)$ & $<0.0001$ \\
\hline $\begin{array}{l}\text { Presence of solid component, } \\
n=62\end{array}$ & $8(57 \%)$ & $12(25 \%)$ & $<0.05$ \\
\hline Mixed/Heterogenous, $n=62$ & $13(92.8 \%)$ & $8(16.7 \%)$ & $<0.001$ \\
\hline Presence of septa, $n=38$ & $6(85.7 \%)$ & $22(70.9 \%)$ & 0.23 \\
\hline Septal Thickness $>3 \mathrm{~mm}, \mathrm{n}=14$ & $3(75 \%)$ & $5(50 \%)$ & 0.02 \\
\hline Ascites, $\mathrm{n}=67$ & $9(64 \%)$ & $3(5.7 \%)$ & $<0.0001$ \\
\hline \multicolumn{4}{|l|}{ Doppler Parameters } \\
\hline $\begin{array}{l}\text { Abundant / Moderate Flow, } \\
\mathrm{n}=22\end{array}$ & $8(89 \%)$ & $1(7.7 \%)$ & $<0.0001$ \\
\hline Central Vascularity, $\mathrm{n}=17$ & $8(100 \%)$ & $0(0.00 \%)$ & $<0.0001$ \\
\hline $\begin{array}{l}\text { Resistance Index(RI) }<0.4, \\
\mathrm{n}=11\end{array}$ & $4(80 \%)$ & $4(67 \%)$ & 0.13 \\
\hline $\begin{array}{l}\text { Peak Systolic Velocity } \\
>15 \mathrm{~cm} / \mathrm{sec}, \mathrm{n}=19\end{array}$ & $7(87 \%)$ & $1(9 \%)$ & $<0.0001$ \\
\hline
\end{tabular}

$\dagger \mathrm{n}$ represents the number of patients in whom the particular parameter was evaluated or data regarding the same was available. $\neq$ percentage positivity in patients with malignant tumor, $\S$ percentage positivity in patients with benign tumor 
TABLE 4: Diagnostic performance of individual ultrasound and Colour Doppler parameters in predicting malignancy

\begin{tabular}{|c|c|c|c|c|}
\hline Ultrasonic Parameters & Sensitivity & Specificity & PPV* & NPV† \\
\hline Tumor Diameter $>5 \mathrm{~cm}$ & $90.48 \%$ & $15.00 \%$ & $21.84 \%$ & $85.71 \%$ \\
\hline Tumor diameter $>10 \mathrm{~cm}$ & $66.7 \%$ & $55.6 \%$ & $28.6 \%$ & $86.2 \%$ \\
\hline Irregular Wall & $100 \%$ & $96.47 \%$ & $84.21 \%$ & $100 \%$ \\
\hline Bilateral Disease & $55 \%$ & $91.14 \%$ & $61.11 \%$ & $88.89 \%$ \\
\hline Presence of solid component & $56.52 \%$ & $75.32 \%$ & $40.63 \%$ & $85.29 \%$ \\
\hline $\begin{array}{l}\text { Mixed/ Heterogenous } \\
\text { echotexture }\end{array}$ & $91.30 \%$ & $83.12 \%$ & $61.76 \%$ & $96.97 \%$ \\
\hline Presence of septa & $84.21 \%$ & $29.27 \%$ & $21.62 \%$ & $88.89 \%$ \\
\hline Septal Thickness $>3 \mathrm{~mm}$ & $75 \%$ & $50 \%$ & $36.84 \%$ & $83.72 \%$ \\
\hline Ascites & $65 \%$ & $94.94 \%$ & $76.47 \%$ & $91.46 \%$ \\
\hline \multicolumn{5}{|l|}{ Doppler parameters } \\
\hline Abundant / Moderate Flow & $87.80 \%$ & $91.67 \%$ & $87.80 \%$ & $91.67 \%$ \\
\hline Central Vascularity & $100 \%$ & $100 \%$ & $100 \%$ & $100 \%$ \\
\hline Resistance Index (RI) $<0.4$ & $80 \%$ & $33.33 \%$ & $50 \%$ & $66.67 \%$ \\
\hline $\begin{array}{l}\text { Peak Systolic Velocity >15 } \\
\mathrm{cm} / \mathrm{sec}\end{array}$ & $87.6 \%$ & $83.4 \%$ & $87.6 \%$ & $83.4 \%$ \\
\hline
\end{tabular}

*PPV: Positive Predictive Value, †NPV: Negative Predictive Value, 
FIGURE 1: Colour Doppler of ovarian mass showing a high peak systolic velocity of 94 $\mathrm{cm} / \mathrm{sec}$. The mass was malignant.

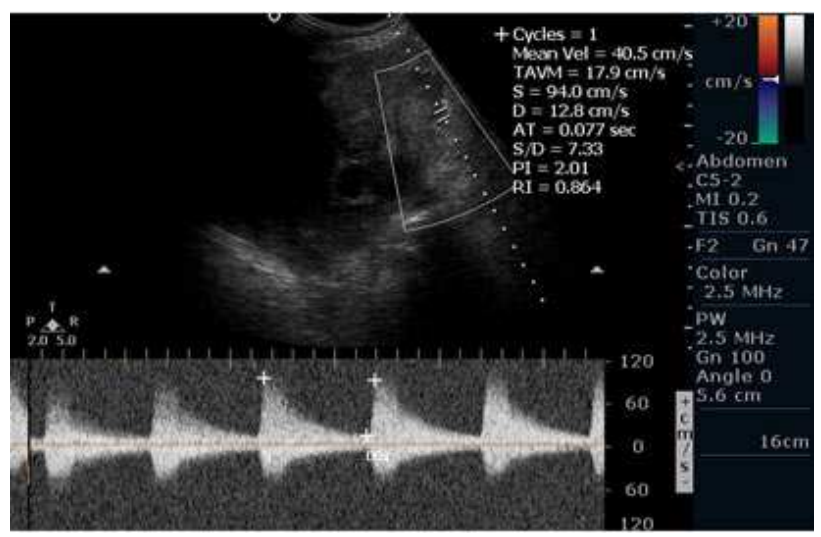

FIGURE 2: Receiver Operator Characteristic (ROC) curve for Alcazar Score in predicting malignancy

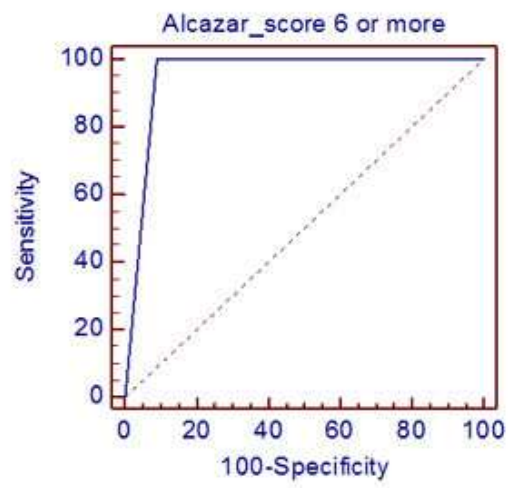

Area under the curve $=0.95$

FIGURE 3: Receiver Operator Characteristic (ROC) curve for Serum CA-125 levels in predicting malignancy

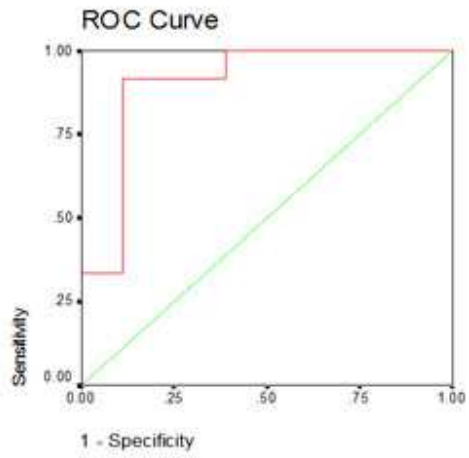

Area under the curve $(\mathrm{AUC})=0.9$ 